\title{
INTERVENCIONES DE ENFERMERIA EN LA PERSONA CON VASOESPASMO CEREBRAL SECUNDARIO A HEMORRAGIA SUBARACNOIDEA ANEURISMÁTICA
}

\begin{abstract}
NURSING INTERVENTIONS IN THE PERSON WITH A SECONDARY CEREBRAL VASOSPASM ANEURYSMAL SUBARACHNOID HEMORRHAGE
\end{abstract}

Recibido marzo 2015 Aceptado mayo 2015

Correspondencia: Lic. Bravo Castrejón Yadira yadypink@hotmail.com

\section{Autores:}

Lic. Yadira Bravo Castrejón

Licenciada en Enfermería, especialista en atención del adulto en estado crítico Adscrita La Unidad de Cuidados Intermedios y Recuperación del Instituto Nacional de Neurología y Neurocirugía Manuel Velasco Suárez, (INNN MVS).

Mtra. Angélica Guadalupe Dávalos Alcázar Maestra en Ciencias de la Educación. Licenciada en Enfermería y Obstetricia, especialista en enfermería neurológica y neuroquirúrgica, adscrita a la coordinación de investigación INNN MVS.

Enf. Neurol. Zoraya Galicia Polo Enfermera especialista en enfermería neurológica y neuroquirúrgica, adscrita al servicio neurocirugía INNN MVS.

Palabras clave: Intervenciones enfermería, vasoespasmo cerebral, hemorragia subaracnoidea, aneurismática.

Key Words: Nursing interventions, cerebral vasospasm, aneurysmal, subarachnoid hemorrhage 


\section{RESUMEN}

Introducción. El vasoespasmo cerebral como complicación de la Hemorragia subaracnoidea aneurismática, es considerado como una vasoconstricción patológica de las arterias principales de la base del encéfalo; es una condición reversible. que se caracteriza con la reducción del calibre de la luz de las arterias y por lo consiguiente una disminución del flujo sanguíneo al área perfundida por el vaso comprometido.

Objetivo. Desarrollar un proceso enfermero basado en la taxonomía Nanda, Noc, Nic a una persona con vasoespasmo cerebral.

Metodología. Se realizó la elección de un caso clínico, con la metodología del proceso de atención de enfermería estableciendo un plan de cuidados en el área de recuperación y terapia intermedia del Instituto Nacional de Neurología y Neurocirugía Manuel Velasco Suárez durante el periodo postauirúrgico inmediato. Se detectaron las necesidades básicas alteradas al realizar la valoración neurológica de enfermería. Se procedió a formular los diagnósticos de enfermería reales y de riesgo y con base en estos se planearon las intervenciones de enfermería.

Conclusión. El realizar las intervenciones de enfermería de forma sistematizada a partir del proceso enfermero, se establece un méłodo científico, en donde cada intervención se fundamenta y da pauta a la aplicación de un cuidado especializado, dirigido hacia la mejora de la persona desde el punto de vista individual y con ello detectar oportunamente signos y síntomas de alarma así como posibles complicaciones.

Palabras clave: intervenciones enfermería, vasoespasmo cerebral, hemorragia subaracnoidea, aneurismática

\section{ABSTRACT}

Introduction. The cerebral vasoespasmo as complication of the Hemorrhage subaracnoidea aneurismática, is considered to be a pathological vasoconstricción of the main arteries of the base of the brain; it is a reversible condition, which is characterized by the reduction of the caliber of the light of the arteries and for consequent a decrease of the blood flow to the area perfundida for the awkward glass.

Target. To develop a process nurse based on the taxonomy Nanda, NOC, NIC to a person with vasoespasmo cerebral.

Methodology. There was realized the election of a clinical case, with the methodology of the process of attention of infirmary establishing a care plan in the field of recovery and intermediate therapy of the National Institute of Neurology and Neurosurgery Manuel Velasco Suárez during the immediate postsurgical period. The basic needs were detected altered on having realized the neurological infirmary evaluation. One proceeded to formulate the real diagnoses of infirmary and of risk and with base in these the infirmary interventions were planned.

Conclusion. Realizing the interventions of infirmary of form systematized from the process nurse, establishes a scientific method, where every intervention is based and gives rule to the application of a specializing care, directed to the progress of the person from the individual point of view and with it to detect opportunely signs and symptoms of alarm as well as possible complication

Key Words: Nursing interventions, cerebral vasospasm, aneurysmal, subarachnoid hemorrhage. 


\section{INTRODUCCIÓN}

巨

n la década de los 60’ del siglo pasado se observó cómo el evento vascular cerebral aumentaba la mortalidad; actualmente con las modificaciones terapéuticas se ha visto mejoría y se han delimitado las complicaciones de esta enfermedad y por lo tanto es imperante que el profesional de enfermería detecte y establezca a partir de los diagnósticos enfermeros; como y cuando debe proceder teniendo las bases teóricas que fundamenten sus intervenciones detectando los signos y síntomas que den directriz en la evolución del enfermo con vasoespasmo cerebral secundario a Hemorragia Subaracnoidea (HSA) Aneurismática.

La realización de un proceso de enfermería es crucial e importante en la atención, ya que es un instrumento de gestión utilizado para el cuidado. El Proceso de Enfermería se define como "el sistema de la práctica de Enfermería, en el sentido de que proporciona el mecanismo por el que el profesional de Enfermería utiliza sus opiniones, conocimientos y habilidades para diagnosticar y tratar la respuesta del cliente a los problemas reales o potenciales de la salud" " y se establecen a razón de la Taxonomía Nanda, Noc, Nic en donde este es el lenguaje del enfermero que unifica el proceder a nivel mundial.

Actualmente, los sistemas de clasificación de lenguaje más conocidos y utilizados en la realidad son las taxonomías NANDA-International (NANDA-I)2, Clasificación de Intervenciones de Enfermería-NIC-3 ${ }^{3}$ y Clasificación de Resultados de Enfermería-NOC-4. Esas clasificaciones favorecen la comunicación y la documentación sistemática de las acciones de la enfermería, además de otros beneficios relacionados a la práctica clínica.

\section{BASES NEUROFISIOPATOLOGICAS}

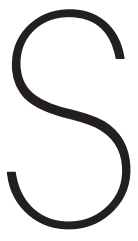

e conoce como enfermedad vascular cerebral a todos los trastornos en los cuales se daña un área del cerebro de forma perma-

nente o transitoria a causa de isquemia cerebral - hemorragia y en los cuales uno o más vasos sanguíneos cerebrales presentan una alteración primaria por algún proceso patológico 5 .

El vasoespasmo cerebral como complicación de la hemorragia subaracnoidea (HSA) aneurismática fue descrito por primera vez por Ecker y Riemenschnaider hace poco más de 50 años ${ }^{\circ}$ Aunque mucho se ha avanzado en el manejo de este evento en las últimas décadas, aún se mantienen aspectos enigmáticos en su comprensión y desafiantes para su control.

En la década de los 60's del siglo XX, la mortalidad registrada fue en un 40 por ciento en la actualidad se ha reportado un descenso hasta del 8 por ciento o menos; este dato se debe en gran medida a la introducción y modificación de las medidas terapéuticas del manejo de los pacientes con vasoespasmo cerebral clínico?. Por lo tanto, el vasoespasmo cerebral es el factor modificable, más importante para mejorar la tasa de morbimortalidad en enfermos con HSA aneurismática y por ende el profesional de enfermería está obligado a definir planes de cuidados especializados que guíen y den directriz a las diversas intervenciones, donde el objetivo sea el identificar oportunamente y de forma imperante el inhibir en lo posible, el curso de esta devastadora complicación.

El vasoespasmo cerebral se considera como una vasoconstricción patológica de las arterias intracraneales principales de la base del encéfalo; es una condición reversible que se caracteriza con una reducción del calibre de la luz de la arteria y por lo consiguiente una disminución del flujo sanguíneo al área perfundida por el vaso comprometido ${ }^{8}$.

104 IMNVMH, vol.14. No. 2 mayo-agosto 2015 
Tipos De Vasoespasmo

Existen tres tipos diferentes de vasoespasmo cerebral, dependiendo del método de detección utilizado: El vasoespasmo angiográfico, sonográfico y sintomático.

La definición del vasoespasmo cerebral sintomático es la de un deterioro neurológico de origen isquémico y diferido en el tiempo.

El vasoespasmo cerebral se clasifica como: leve, moderado y grave; usualmente inicia del día $3^{\circ}$ al 5 de la HSA, se observa la mayor estrechez de la luz arterial desde el día 5ㅇ al día 7ㅇ, y se prolonga en promedio hasta el día 14\%; se resuelve en el curso de 2 a 4 semanas?. Por lo tanto el profesional de enfermería deberá tener énfasis en la valoración neurológica en este periodo ya que es considerado como crítico.

Los factores de riesgo más importantes son la hipertensión, la enfermedad cardiaca, el uso de cocaína y el consumo de cigarrillos ${ }^{10}$. Otros factores incluyen el elevado consumo de alcohol y niveles altos de colesterol en la sangre.

\section{ETIOLOGÍA}

$H$ asta el día de hoy no está claramente definida, sin embargo el estrechamiento de la luz del vaso puede causar aumento de la resistencia vascular y con ello la disminución del flujo sanguíneo cerebral a niveles críticos que puede causar isquemia y lesión cerebral.

Se han sugerido varios factores, incluyendo productos de degradación de la hemoglobina, mediadores inflamatorios e hiperreactividad de los músculos lisos cerebrovasculares y de los núcleos catecolaminérgicos del tronco neurológico.

\section{EPIDEMIOLOGÍA}

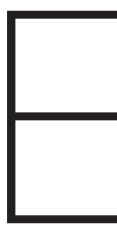
n general la enfermedad vascular cerebral (EVC) constituye una de las principales causas de muerte y la principal causa de incapacidad en sujetos mayores de 60 $a^{n} \operatorname{ss}^{10}$.

Los eventos hemorrágicos constituyen el 20 por ciento del total de los eventos cerebrovasculares, resultando la localización subaracnoidea la más frecuente por encima del 50 por ciento y esta a su vez es multifactorial, siendo los aneurismas intracraneales los más frecuentes del 21 al 72 por ciento ${ }^{12}$.

La Hemorragia Subaracnoidea (HSA) es una enfermedad común y en algunos casos devastadora, se ha demostrado 25 por ciento de mortalidad y el pronóstico depende del estado de conciencia en el que se encuentre el paciente al inicio del tratamiento ${ }^{13}$.

La Hemorragia subaracnoidea (HSA) aneurismática es una importante causa de muerte prematura, la cual ocurre con mayor frecuencia entre los 40 y 60 años de edad. Con relación al comportamiento en cuanto al género presenta una incidencia superior en las mujeres con un promedio del 54-67 por ciento ${ }^{14}$.

El vasoespasmo por HSA aneurismática, es una complicación frecuente encontrada hasta en un 70\% de los pacientes que presentan esta enfermedad; estos presentan daño isquémico hasta un 20\%, y aumenta la mortalidad en estos pacientes que presentan esta complicación, por lo cual es importante ofrecer tratamiento preventivo y terapéutico oportuno ${ }^{15,16}$. 


\section{SIGNOS Y SÍNTOMAS}

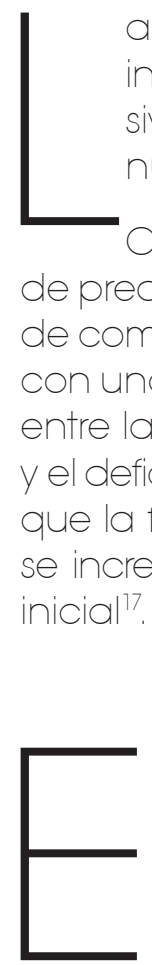

a sintomatología característica es cefalea intensa, nausea, vomito, alteración progresiva del estado de conciencia, rigidez de nuca, déficit motriz.

Otro medio que se ha utilizado como factor de predicción de vasoespasmo es el grado clínico de compromiso neurológico del paciente admitido con una HSA; existe además una clara correlación entre la evidencia angiográfica de vasoespasmo y el deficiente estado clínico de ingreso; esto significa que la frecuencia y la gravedad del vasoespasmo se incrementan con el deterioro del estado clínico inicial ${ }^{17}$.

\section{DIAGNÓSTICO MÉDICO}

tado por un deterioro de del estado de conciencia. afasia, déficit motor ${ }^{78}$.

El soporte en los estudios neurorradiológicos es de gran beneficio para determinar el riesgo de vasoespasmo cerebral, caracterizar la localización del aneurisma, establecer el plan de intervención quirúrgica, monitoreo del paciente y así descartar el desarrollo de vasoespasmo o de diagnosticarlo precozmente, todo esto con el propósito de optimizar la terapia de intervención.

Entre los estudios a realizar se encuentran principalmente la Angiografía cerebral, Doppler transcraneal y la angiotomografía computarizada ${ }^{19,20}$

La optimización del estado hemodinámico del paciente puede ser lograda mediante la aplicación de la llamada terapia triple H (hipertensión, hemodilución e hipervolemia).
El trastorno de la autorregulación cerebral en el área de penumbra isquémica, determina que el flujo en esta zona dependa directamente de la presión de perfusión cerebral (PFC) que puede ser mejorada mediante al aumento de la presión arterial media (PAM) o reducción de la presión intracraneal (PIC).

No obstante, aunque la efectividad de este método ha sido casi siempre anecdótica, dichos reportes son suficientemente convincentes para mantener su aplicación actual.

Entre los medicamentos de elección están fármacos calcio-antagonistas como es el nimodipino, se ha demostrado mejorar el pronóstico funcional, sin que se haya observado que pueda reducir la aparición radiológica de vasoespasmo. Este beneficio se asume que es debido a su efecto neuroprotector vascular. A la inversa, el nicardipino ha demostrado reducir un 30 por ciento las cifras de vasoespasmo, sin que por contra redunde en un beneficio funcional para los pacientes22.

El tratamiento también incluye medidas anti edema cerebral, así como protectores de la mucosa gástrica, sin olvidarnos de la intervención de la clínica del dolor, y ablandadores de heces.

El tratamiento endovascular consiste en la angioplastia con balón y la infusión intraarterial de papaverina.

La infusión intravenosa de vasodilatadores produce hipotensión antes de que las concentraciones sean suficientemente altas como para dilatar las arterias vasoespásticas.

Otros fármacos vasodilatadores se han usado de forma anecó́tica para la irrigación intraarterial de la circulación cerebral en el tratamiento del vasoespasmo sintomático secundario a HSA aneurismática como son el nimodipina, verapamilo, fasudilo ${ }^{23}$.

\section{6}

NWMm vol.74. No. 2 mayo - agosto 2075 


\section{PRESENTACIÓN DEL CASO}

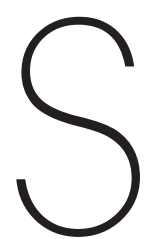
e trata de una fémina de 52 años de edad, soltera y de religión católica; de profesión científica e intelectual, originaria de la Ciudad de México, actualmente reside en el Estado de Morelos. Vive sola en casa medio urbana que cuenta con todos los servicios intradomiciliarios. Sus hábitos higiénicos y dietéticos son adecuados en calidad y cantidad. Como antecedentes familiares tiene hipertensión arterial sistémica, y enfisema pulmonar; y presenta antecedentes personales patológicos de alergia a la penicilina. Su factor de riesgo es el tabaquismo desde los 16 años de edad, consumiendo a razón de 10 cigarrillos al día.

Inicio su padecimiento el 23 de diciembre del 2014 al ingerir alimentos, manifiesta cefalea intensa 9/10 (escala de EVA) con pérdida del estado de alerta, presencia de crisis convulsivas y vomito de contenido gastro- alimenticio.

Se traslada a nosocomio del Estado de Morelos donde presenta afasia motora, solo sigue ordenes sencillas y presenta descontrol glicémico.

El 3 de enero del 2015 es valorada en el servicio de urgencias del Instituto Nacional de Neurología y
Neurocirugía Manuel Velasco Suárez (INNN MVS) donde se encuentra despierta, inatenta, sin emisión de lenguaje, no obedece órdenes sencillas, no cooperadora con pupilas isométricas, de $3 \mathrm{mms}$.

A su ingreso al (INNN MVS) se realizó el diagnóstico de Hemorragia subaracnoidea (HSA), con escala Fisher IV y Hunt y Hess IV por probable ruptura de aneurisma en arteria cerebral media izquierda e hidrocefalia aguda, confirmando el diagnostico mediante angiografía y tomografía axial computarizada.

Se realiza Clipaje de aneurisma de arteria cerebral media (ACM ) izquierda y Derivación ventrículo peritoneal el día 6 de enero y ya instalada en el área post-quirúrgica se presenta con herida quirúrgica en región fronto temporal izquierda, inatenta con tendencia a la somnolencia, con pupilas isométricas, normorreflécticas hemiparesia derecha, con agitación psicomotriz, por lo que requiere contención física; continua con afasia motora, reflejo nauseoso y deglución ausente, con una sonda nasogástrica para apoyo nutricio y farmacológico; con apoyo de oxigeno suplementario a través de tienda facial

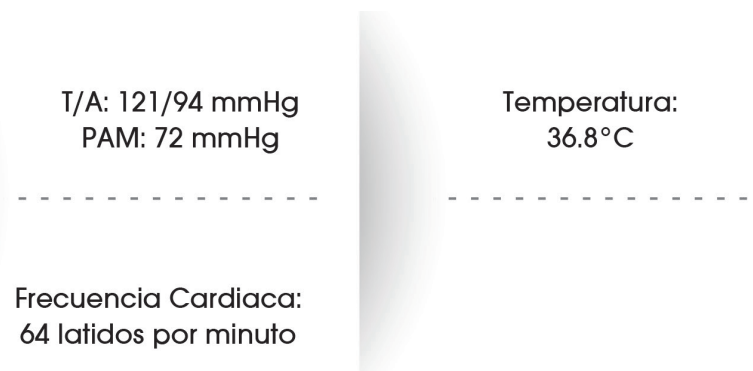

\section{Presión venosa central $8 \mathrm{~cm}$ de H2O}

- . . . . . . . . .

Frecuencia respiratoria: 24 respiraciones por minuto.

Se ministra soporte hemodinámico a través de fármacos vasopresores (vasopresina y norepinefrina) para mantener una Tensión Arterial Media (TAM) 100-110 mmHg. Y con manejo de terapia triple H. a través de catéter central subclavio derecho para mantener parámetros hemodinámicos óptimos. 
A partir de la valoración neurológica de enfermería y jerarquización de necesidades se procede y se establecen los diagnósticos post quirúrgicos reales y de riesgo siendo los siguientes:

NOTA: Por cuestión editorial se enumeran los referentes, enfocándonos al de relevancia inmediata.

\section{DIAGNOSTICOS REALES}

Diagnóstico
Perfusión tisular cerebral ineficaz.
Deterioro de la comunicación verbal.
Ansiedad.
Confusión aguda.
Alteración de la la percepción
Deterioro de la deglididad física.

\section{Diagnóstico}

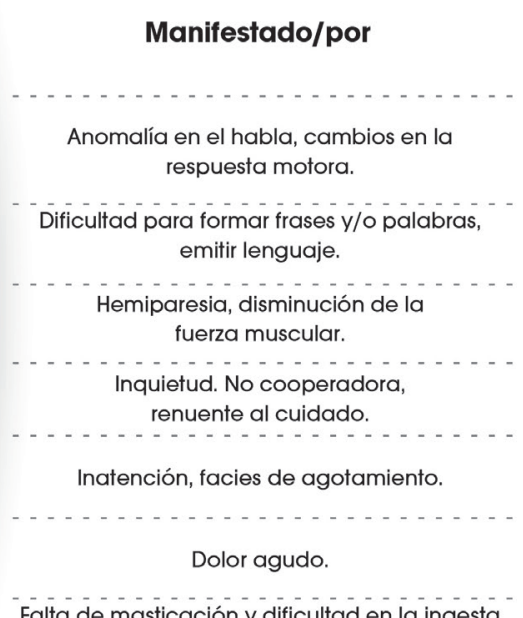

Falta de masticación y dificultad en la ingesta de sus medicamentos.

\section{DIAGNÓSTICOS DE RIESGO}

\section{Diagnóstico}

Riesgo de caída.

Riesgo de desequilibrio de volumen de líquidos.

Riesgo de desequilibrio electrolítico.
Relacionado/con

Alteración del estado mental y disminución de la fuerza.

Abundante aporte de líquidos.

Deterioro de los mecanismos reguladores. 


\section{PLAN DE CUIDADO}

Dominio: 4 actividad/reposo
Clase: 4 respuestas cardiovasculares / pulmonares

Dx. Enfermería

Perfusión tisular cerebral ineficaz r/c interrupción del flujo arterial o venoso. $\mathrm{m} / \mathrm{p}$ anomalía en el habla, cambios en la respuesta motora, alteración del estado mental.

\section{Concepto: patron cardiovascular}

Sujeto: no

Juicio: ineficaz

Localización: cerebral

Edad: Adulto

Definición Adecuación del flujo sanguíneo a través de las arterias cerebrales para mantener la función cerebral.

Tiempo: Posquirúrgico inmediato

Resultado esperado: Perfusión tisular cerebral.

Estado: Real

\begin{tabular}{|l|}
\hline INDICADORES \\
\hline Presión de perfusion cerebral \\
\hline Presion arterial media \\
\hline
\end{tabular}

Presion intracraneal

INTERVENCIONES: Mejora de la perfusión cerebral.

Definieion: Fomento de una perfusión adecuada y limitación de complicaciones en una persona que experimenta o corre el riesgo de experimentar una perfusión cerebral incorrecta.

\section{AGTIVIDADES}

Inducir la hipertensión arterial con la expansión del volumen o con agentes inotrópicos o vasoconstrictores, según prescripción para mantener los parámetros hemodinámicos y mejorar la presión de perfusión cerebral (PPC).

Administrar agentes que expandan el volumen intravascular, si procede, (coloides, productos sanguíneos y cristaloides).

Mantener en nivel de hematocrito alrededor del $33 \%$ para la terapia de hemodilución hipervolémica.

Mantener la posición óptima de la cabecera de la cama $30^{\circ}$

Ministrar analgesia si procede.

Mantener el nivel de glucosa en suero dentro del margen normal.

Gravemente comprometido 1

Levemente comprometido 4
Dominio: salud fisiologica (II)

Clase: Cardiovascular (E)

ESCALA

Sustacialmente comprometido 2

Moderadamente comprometido 3

No comprometido 5
Fundamentación

La presión arterial media es el promedio de la presión de la sangre en las arterias a lo largo del ciclo cardiaco, es decir, la presión sostenida que se mantiene dentro de los vasos sanguíneos dependiendo de los niveles de presión sistólica y diastólica; este es un valor que indica la presión que debe tener el corazón, teniendo un flujo constante , para mantener una adecuada perfusión tisular; se asume que la PAM es un indicador importante del flujo sanguíneo arterial orgánico ${ }^{24}$, sin embargo, una de las limitaciones que presenta es que en situaciones de hipotensión, los barorreceptores carotideos alteran el tono vascular de forma que aumenta la presión arterial y por lo tanto la PAM se mantiene, mientras que el flujo de sangre y el transporte de oxigeno a algunos órganos puede ser inadecuado ${ }^{25}$. Los monitores calculan la presión arterial media, aunque este cálculo lo puede realizar el enfermero, teniendo en cuenta que la irrigación de los tejidos se da en un tercio de la sístole y en dos tercios de la diástole. Con base a lo anterior se utiliza la siguiente ecuación para el cálculo de la presión arterial media ${ }^{26}$.

\section{PAM: 2PAD + PAS}

PAD: Presión arterial diastólica.

PAS: Presión arterial sistólica.

Para mantener una perfusión orgánica adecuada es necesario que la presión arterial media se encuentre sobre $65 \mathrm{mmHg}$, aunque para decir que el paciente cursa por un periodo de mala perfusión se requiere llevar un seguimiento de estos valores y relacionarlos con otros parámetros hemodinámicos y clínicos como frecuencia cardiaca, presión venosa central, gasto urinario, estado de conciencia, color de la piel y llenado capilar. Cuando hay un aumento en la demanda metabólica del organismo, se incrementa el flujo sanguíneo a los órganos por vasodilatación local de las pequeñas arteriolas. Si el volumen minuto cardíaco no puede incrementarse de manera adecuada, la presión arterial disminuye. Si la demanda metabólica se mantiene constante, se producen cambios en el tono de las paredes arteriales lo que produce un aumento de la presión arterial para mantener el flujo sanguineo orgánico constante. Este mecanismo de control vasomotor local se denomina autorregulación. Si bien la autorregulación ocurre en órganos como el cerebro, el hígado, el músculo esquelético y la piel, no es un fenómeno universal ${ }^{27.28 .}$ Una presión arterial normal no significa que todos los órganos tengan una perfusión adecuada porque el aumento del tono vasomotor local y las obstrucciones mecánicas pueden aún inducir isquemia vascular asimétrica. Sin embargo, la hipotensión es siempre una alteración que se asocia con disminución de la perfusión orgánica. ${ }^{29,30}$ 


\begin{tabular}{|c|c|}
\hline INTERVENCIONES : Monitoreo de la presion arterial media. & Fundamentacion \\
\hline $\begin{array}{l}\text { Definicion: Medicion e interpretacion de parametros de la PAM } \\
\text { invasiva para determinar la funcion cardiovascular para } \\
\text { determinar y prevenir complicaciones. }\end{array}$ & \multirow{7}{*}{$\begin{array}{l}\text { El monitoreo invasivo de la PAM requiere del uso de dispositivos que } \\
\text { son insertados en algunos de los vasos sanguíneos del sistema } \\
\text { cardiovascular con el fin de oblener mediciones de las presiones } \\
\text { que se generan dentro de estos. Para realizar esta clase de moni- } \\
\text { toreo se requiere atravesar una de las barreras naturales del cuerpo } \\
\text { como la piel, lo que puede aumentar el riesgo de desarrollar } \\
\text { procesos infecciosos } \text { s. }^{31} \text { La correcta monitorizacion invasiva permite } \\
\text { llevar un control continuo y se utiliza en los pacientes que se } \\
\text { encuentran hemodinamicamente inestables o que presentan una } \\
\text { alteración en el organismo que pueden llevar a un mal funciona- } \\
\text { miento del sistema cardiovascular }{ }^{32}\end{array}$} \\
\hline Realizar la insercion de una linea hemodinamica invasiva. & \\
\hline $\begin{array}{l}\text { Poner a cero y calibrar el equipo cada } 4 \text { horas si procede, } \\
\text { con el transductor a nivel de la auricula derecha. }\end{array}$ & \\
\hline $\begin{array}{l}\text { Comparar los parametros hemodinámicos con los signos y } \\
\text { sintomas clínicos. }\end{array}$ & \\
\hline Monitorizar la perfusión periférica distal al sitio de insercion. & \\
\hline $\begin{array}{l}\text { Mantener la esterilidad de los orificios asi como el circuito } \\
\text { cerrado del sistema. } \\
\text { Cambiar la solucion heparinizada y los sistemas o equipos } \\
\text { cada } 24 \text { horas si procede, según protocolo. }\end{array}$ & \\
\hline $\begin{array}{l}\text { Administrar liquidos y/o expansores de volumen para mantener } \\
\text { el parametro de la PAM dentro del margen especificado. } \\
\text { Administrar agentes farmacologicos para mantener la PAM } \\
\text { dentro del margen especificado. } \\
\text { Instruir al paciente sobre la restriccion de actividades mientras la } \\
\text { linea arterial permanece en su sitio. }\end{array}$ & \\
\hline INTERVENCIONES: Presion intracraneal. & Fundamentacion \\
\hline $\begin{array}{l}\text { Definicion: La Presión Intracraneal, se define como aquella } \\
\text { medida en el interior de la cavidad craneal que es el resultado } \\
\text { de la interacción entre el continente (cráneo) y el contenido } \\
\text { (encéfalo, LCR y sangre). }\end{array}$ & \multirow{7}{*}{$\begin{array}{l}\text { La doctrina de Monro-Kellie establecía que el cráneo es un com- } \\
\text { partimento rigido con tres componentes no compresibles: tejido } \\
\text { cerebral, volumen sanguíneo cerebral y líquido cefalorraquídeo, y } \\
\text { que un incremento de la PIC puede originar un descenso de la PPC, } \\
\text { lo cual conlleva isquemia cerebral y un peor pronóstico } \\
\text { Una vez la presión intracraneal ha sido determinada, es fácil obtener } \\
\text { la presión de perfusión cerebral (PPC), la cual está dada por la } \\
\text { diferencia entre la presión arterial media y la presión intracraneal. } \\
\text { (PPC= PAM-PIC). Esta presión debe mantenerse como mínimo en } \\
70 \text { mmHg. }^{35}\end{array}$} \\
\hline Monitorizacion de la presion intracraneal (PIC). & \\
\hline $\begin{array}{l}\text { Poner a cero y calibrar el equipo cada } 4 \text { horas si procede, con } \\
\text { el fransductor. }\end{array}$ & \\
\hline Registrar la caracteristica y forma de la onda de PIC. & \\
\hline Registrar la lectura de presion de la PIC. & \\
\hline $\begin{array}{l}\text { Vigilar el estado neurologico. } \\
\text { Observar la frecuencia, cantidad y caracterisiticas del liquido } \\
\text { cefalorraquideo (LCR). } \\
\text { Prevenir el desplazamiento del dispositivo de monitorizacion. } \\
\text { Mantener esteril el sistema de monitoreo y drenaje. }\end{array}$ & \\
\hline $\begin{array}{l}\text { Obtener muestras del drenaje de LCR. } \\
\text { Observar el sitio de insercion. } \\
\text { Evitar maniobras de Valsalva. }\end{array}$ & \\
\hline EVALUACIÓN & $\begin{array}{l}\text { Las intervenciones realizadas fueron asertivas, sin embargo no se } \\
\text { logró mantener la presión arterial media deseada ya que la paciente } \\
\text { presentó disfunción del sistema nervioso autónomo y por lo tanto } \\
\text { no tuvo una respuesta adecuada a los vasopresores. }\end{array}$ \\
\hline
\end{tabular}

El día 7 de enero se realizó Angiografía cerebral donde se reportó Vasoespasmo multifocal moderado de la arteria cerebral media izquierda con respuesta parcial a la administración de nimodipino intraarterial por lo que se decide trasladar a la Unidad de Terapia Intensiva para continuar manejo.

\section{0}

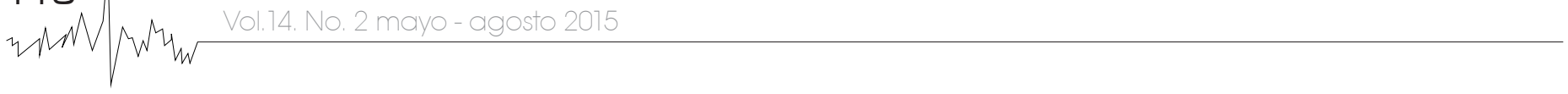




\section{CONCLUSION}

巨

I realizar las intervenciones de enfermería de forma sistematizada a partir del proceso enfermero, establece un método científico en donde cada intervención se fundamenta y da pauta a la aplicación de un cuidado especializado, dirigido hacia la mejora de la persona desde el punto de vista individual y con ello detectar oportunamente signos y síntomas de alarma así como posibles complicaciones.

Al hacer referencia a la utilización del proceso enfermero, da estructura al caso clínico nos va direccionando en la valoración de la persona mediante la recolección de diversos datos que posteriormente jerarquizan las necesidades humanas, con ello diagnosticamos y planeamos el cuidado, a través de esta planificación se fundamenta científicamente el proceder del profesional enfermero, sustentando los conocimientos de la propia patología como del mismo proceso de enfermedad y las condiciones del enfermo.

A partir de éste momento en que es nuestra responsabilidad, mentalmente estructuramos el proceder profesional, a bien de contar con un bagaje cultural y científico propio, dado por la formación académica, en este caso se diagnosticaron y dieron las intervenciones ex profeso; sin embargo la persona necesito cuidado intensivo, por su condición hemodinámica por lo que fue trasladada de la unidad de recuperación (área post quirúrgica) a la unidad de terapia intensiva, por ello se establece el límite de nuestra intervención, sin embargo es imperante dar la información al realizar la entrega y recepción de la persona a otro profesional enfermero, en este caso en área crítica, enfatizando la valoración inicial y como fue evolucionando así como las intervenciones independientes como interdependientes, lo que determina dar continuidad de cuidado evitando en el paciente riesgos y daños que pudieran poner en riesgo su vida.

\section{REFERENCIAS BIBLIOGRÁFICAS}

1. 2P. Iyer, B. Taptich, y D. Bernocchi. Proceso de enfermería y diagnósticos de enfermería. 3ra edición. Mc Graw -Hill. 1997. p. 11 disponible en: http://www.redalyc.org/pdf/304/304212940 03.pdf

2. NANDA - International. Nursing diagnoses: definitions \& classification 2012-2014. Philadelphia: Wiley-Blackwell; 2012. 568 p. Disponible en: http://www.scielo.br/scielo.php?script=sci_nlinks\&ref= 000082\&pid=S0104- $1769201200060001300002 \&$ Ing=en

3. Bulechek GM, Butcher HK, Dochterman JM. Classificação das intervenções de enfermagem - NIC. 5" ed. Rio de Janeiro: Elsevier; 2010. 944 p.Disponible en: http://www.scielo.br/scielo.php?script=sci_nlinks\&ref=000086\&pid=S0104- $7169201200060001300004 \&$ Ing $=e n$

4. Moorhead S, Johnson M, Maas M. Classificação dos resultados de enfermagem - NOC 4"ed. Rio de Janeiro: Elsevier; 2010. 936 p. Disponible en http://www.scielo.br/scielo.php?script=sci_nlinks\&ref= 000086\&pid=S0 104- $1769201200060001300004 \&$ Ing=en

5. Aburto Murrieta Yolanda, Araúz Góngora Antonio, Murillo Bonilla Luis Manuel. Factores de riesgo asociados a EVC en joven con foramen oval permeable. Arch. Neurocien. (Mex., D.F.) (revista en la Internet). 2004 Dic (citado 2015 Abr 28) ; 9( 4 ): 206-214. Disponible en: http://www.scielo.org.mx/scielo.php?script=sci_arttext\&pid=S0187-4705200400 1200005\&/ng=es

6. Varela Hernández A, Vega Basulto S, Revista Mexicana de Neurociencia, Vasoespasmo cerebral. Un reto para la medicina moderna. México, D.F: Medigraphic Artemisa. Núm. 6.2005 p491. Disponible en: http://www.medigraphic.com/pdfs/revmexneu/ rmn-2005/rmn056e.pdf

7. Varela Hernández A, Vega Basulto S, Revista Mexicana de Neurociencia, Vasoespasmo cerebral. Un reto para la medicina moderna. México, D.F: Medigraphic Artemisa. Núm. 6.2005 p491 Disponible en: http://www.medigraphic.com/pdfs/revmexneu/rmn2005/rmn056e.pdf

8. Hoh B, Carter B, Ogilvy C. Risk of hemorrhage of unsecured, un ruptured aneurysms during and after hypertensive, hypervolemic therapy. Neurosurgery. 2002; 50:1207-1212. Disponible en: http:// journals.Iww.com/neurosurgery/Abstract/2002/06000/Risk_of_Hemorrhage_from_Unsecured,_Unruptured.6.aspx, Consultado en: http:// $w w w$.scielo.org.co/scielo.php?script=sci_nlinks\&ref=000305\&pid=SO 1 $203347200700020000600008 \&$ Ing=en

9. Mejía C Juan A, Niño de Mejía María C, Ferrer Z Leopoldo E, Cohen M Darwin. Vasoespasmo cerebral secundario a hemorragia subaracnoidea por ruptura de aneurisma intracerebral. Rev. colomb. anestesiol. (serial on the Internet). 2007 Apr (cited 2015 Apr 28) ; 35( 2 ): 143-162. Available from: http://www.scielo.org.co/ scielo. php? script=sci_arttext\&pid=S0 120-33472007000200006\&lng=en

10. Lagares A., Gómez P. A. , Alén J.F., Arikan F., Sarabia R. , Horcajadas A. et al . Hemorragia subaracnoidea aneurismática: guía de tratamiento del Grupo de Patología Vascular de la Sociedad Española de Neurocirugía. Neurocirugía(revista en la Internet). 2011 Abr (citado 2015 Abr 28) ; 22(2): 93-115. Disponible en: http://scielo.isciii. es/scielo.php?script=sci_arttext\&pid=S1 130-147320 11000200001 \&lng=es 


\section{REFERENCIAS BIBLIOGRÁFICAS}

11. Yolanda Aburto Murrieta, Antonio Araúz Góngora, Luis Manuel Murillo Bonilla Archivos de Neurociencias. Mex., D.F. v.9 n.4 México dic. 2004 versión impresa ISSN 0187-4705 consultado http://WwW. scielo.org. $\mathrm{mx} /$ scielo.php?pid=S0187-47052004001200005\&script=sci arttext08/marzo./2014. 20:00

12. Cardentey Pereda. A.L. Pérez Falero R: A. Subarachnoid Hemorrhage. Revista de Neurología. Consultado http://www.revneurol. com/sec/resumen.php?id=2000059 08/marzo/2014. 18:30.

13. Burgos de la E.R. y Díaz Orduz. R. Hemorragia Subaracnoidea Espontánea:Consultado http://med.javeriana.edu.co/publi/vniversitas/serial/v43n4/0057\%20HSE.PDF09/marzo/2014. 20:35

14. IDEM 3. 22p.

15. Carrillo $R$, Leal $P$, Arellano R. Hemorragia subaracnoidea secundaria a ruptura de aneurisma. Medigraphic 2009: 16(1): 10-8. Consultado en: http://www.medigraphic.com/pdfs/finlay/fi-2014/ fi $743 d . p d f$

16. Rose J, Mayer S. Optimizing blood pressure in neurological emergencies. Neurocritic Care 2004; 3:287-99. consultado en :http://www.ncbi.n/m.nih.gov/pubmed/16174926

17. Pickard J, Murray G, Illingworth R, et al. Effect of oral nimodipine on cerebral infarction and outcome after subranoid hemorrhage: British aneurysm nimodipine trial. BMJ. 1989; 298: 636-42.consultado en: http://www.ncbi.nlm.nih.gov/pubmed?cmd=Link\&dbFrom=PubMed\&from_uid=2662635

\section{8. $D E M 636-43$}

19. Revista de Neurología. Utilidad clínica del Doppler transcraneal en el diagnóstico del vasoespasmo cerebral en la hemorragia subaracnoidea. 2008; 47 (6): 295-298 consultado en: http://cat.inist. $\mathrm{fr} /$ ?aModele=afficheN\&cpsidt=20802775

20. Juan A. Mejía C. María C. Niño de Mejía. Eł al Revista Colombiana de Anestesiología. Vasoespasmo cerebral secundario a hemorragia subaracnoidea por ruptura de aneurisma intracerebral vol.35 no.2 Bogotá Jan. /Apr. 2007.consultado en: http://www.scielo.org. co/scielo.php?script=sci_arttext\&pid=S0120-33472007000200006

21. Allen GS, Ahn HS, Preziosi TJ, Battye R, Boone SC, Chou SN, et al. Cerebral arterial spasm: a controlled trial of nimodipine in patients with subarachnoid hemorrhage. N Engl J Med.1983; 308:619fl24. Consultado en: http://stroke.ahajournals.org/content/19/1/63.full.pdf

22. Haley EC, Kassell NF, Torner JC. A randomized controlled trial of high-dose intravenous nicardipine in aneurysmal subarachnoid Hemorrhage: a report of the Cooperative Aneurysm Study. J Neurosurg. 1993;78:537fl47 consultado en: https://ispub.com/IJA/2/2/7173

23. Pedro P. Alcázar, Alejandro González y Antonio Romance. Medicina Intensiva. Tratamiento endovascular del vasospasmo cerebral inducido por hemorragia subaracnoidea aneurismática v.32 n.8 Madrid nov. 2008.consultado en: http://scielo.isciilies/scielo.php?script=sci_anttext\&pid=S02 10-56912008000800004

24. Newberry L, Nanda, Emergency Nurses Association, Sheehy S, Center for Nursing Classilication and Clinical Effectiveness. Manual de urgencia de enfermería: Diagnósticos enfermeros, resultados e intervenciones. España Elsevier. 2007. p. 187. Consultado en: http:// tesis.udea.edu.co/dspace/bitstream/10495/1355/1/Monitoria\%20 Hemodinamica\%20en\%20el\%20Cuidado\%20de\%20Enfermeria\%20 del\%20Paciente\%20con\%

25. Álvar Net e Vallverdú I. Función cardiovascular en el paciente grave. España; Elsevier, 2005. p. 178. Consultado en: http://tesis udea.edu.co/dspace/bitstream/10495/1355/1/Monitoria\%20Hemodinamica\%20en\%20el\%20 Cuidado\%20de\%20Enfermeria\%20del\%20 Paciente\%20con\%

16. Mathew N., Koeppen B, Stanton B, Berne R. Fisiología de Berne Y Levy. España: Elsevier; 2006. p. 290. Consultado en: http://tesis udea.edu.co/dspace/bitstream/10495/1355/1/Monitoria\%20Hemodinamica\%20en\%20el\%20 Cuidado\%20de\%20Enfermeria\%20del\%20 Paciente\%20con\%20

27. Sánchez R. Atención Especializada de Enfermería al Paciente Ingresado en Cuidados Intensivos. Editorial CEP. 2007. p. 92 consultado en: http://tesis.udea.edu.co/dspace/bitstream/10495/1355/1/ Monitoria\%20Hemodinamica\%20en\%20el\%20Cuidado\%20de\%20Enfermeria\%20del\%20Paciente\%20con\%

28. Chatterjee K. The Swan-Ganz Catheters: Past, Present, and Future A Viewpoint. Circulation. 2009; 119: 147-52. Consultado en http://www.ncbi.n/m.nih.gov/pubmed/19124674

\section{IDEM. Consultado en: http://www.ncbi.n/m.nih.gov/pubmed/19124674}

30. Duque Ortiz, Camilo. Monitoria hemodinámica en el cuidado de enfermería de los pacientes en estado crítico con perfusión tisular inefectiva. Diss. 2008. Consultado en: http://tesis.udea.edu.co/ aspace/bitstream/10495/1355/1/Monitoria\%2OHemodinamica\%20en\%20 el\%20Cuidado\%20de\%20Enfermeria\%20del\%20Paciente\%20con\%

31. Mathew N., Koeppen B, Stanton B, Berne R. Fisiología de Berne Y Levy. España: Elsevier; 2006. p. 290.

32. Turchetto E. A qué llamamos paciente críticamente enfermo y cómo lo reconocemos? Rev. Hosp. Priv. Comun. 2005; 8(2):52- 7. Consultado en: http://tesis.udea.edu.co/dspace/bitstream/10495/1355/1/ Monitoria\%20Hemodinamica\%20en\%20el\%20Cuidado\%20de\%20Enfermeria\%20del\%20Paciente\%20con\%20Perfusion\%2

Turchetto E. A qué llamamos paciente críticamente enfermo y cómo lo reconocemos? Rev. Hosp. Priv. Comun. 2005; 8(2):52- Consultado en: http://tesis.udea.edu.co/dspace/bitstream/10495/1355/1/ Monitoria\%20Hemodinamica\%20en\%20el\%20Cuidado\%20de\%20Enfermeria\%20del\%20Paciente\%20con\%20Perfu

33. Carmona, J.V., Lamarca, M.C., Leiva, S., Llabata, P. Gómez, B. Cuidados de enfermería en el paciente con elevación de la PIC. eNFeRMER`A INTEGRAL / Marzo 2006. Pág 3-5. Consultado en: http://www.enfervalencia.org/ei/73/articulos-cientificos/ac_7.pdf

34. Miller JD. Head injury and brain ischemia--implications for therapy. Br J Anaesth. 1985;57:120-30.consultado en: http://www.ncbi. n/m.nih.gov/pubmed/3887770

35. Carmona Simarro JV, Fariña Almor C. Monitorización del metabolismo cerebral; SjO2. Conceptos y cuidados de Enfermería. Enfermería Integral. 2001;59:39-42, consultado en http// www.enfermeriadeurgencias.com/ciber/enero2022/pagina7.html
112 